\title{
Anti-Inflammatory Dietary Approach to Prevent the Development and Progression of Non-Alcoholic Fatty Liver Diseases
}

\author{
Dorothea Portius (D)
}

check for updates

Citation: Portius, D. AntiInflammatory Dietary Approach to Prevent the Development and Progression of Non-Alcoholic Fatty Liver Diseases. Livers 2022, 2, 78-84 https://doi.org/10.3390/ livers2010005

Academic Editor: Hua Wang

Received: 13 December 2021 Accepted: 16 February 2022 Published: 2 March 2022

Publisher's Note: MDPI stays neutral with regard to jurisdictional claims in published maps and institutional affiliations.

Copyright: (C) 2022 by the author. Licensee MDPI, Basel, Switzerland. This article is an open access article distributed under the terms and conditions of the Creative Commons Attribution (CC BY) license (https:// creativecommons.org/licenses/by/ $4.0 /)$.
Nutrition Therapy and Counseling, SRH University of Applied Health Science, 07548 Gera, Germany; dorothea.portius@srh.de

\begin{abstract}
Non-alcoholic fatty liver disease (NAFLD) is an increasing health problem worldwide and is associated with insulin resistance, increased visceral fat mass, and cardiovascular problems. Lifestyle factors such as sedentary lifestyle, chronic stress, obesogenic environment as well as a Western pattern diet are main contributors to the development and progression of this disease. In particular, the diet plays a pivotal role. An unhealthy diet including high consumption of red and processed meats, refined carbohydrates, simple sugars, highly processed foods with food additives and conservatives are lighting the fire for a low-grade inflammation. If other risk factors come into play, metabolic and hormonal derangement may occur, leading to the increase in visceral fat, gut dysbiosis and leaky gut, which stoke the inflammatory fire. Thus, lifestyle interventions are the most effective approach to quell the inflammatory processes. An anti-inflammatory and low-glycemic diet named the GLykLich diet, which includes whole and unprocessed foods, may reduce the risk of increased morbidity and mortality. The GLykLich diet suggests a meal consisting of complex carbohydrates (fiber), good quality of protein and healthy fats (DHA/EPA), and is rich in secondary plant products. There is no single nutrient to prevent the progression of NAFLD, rather, it is the complexity of substances in whole unprocessed foods that reduce the inflammatory process, improve metabolic state, and thus reverse NAFLD.
\end{abstract}

Keywords: anti-inflammatory diet; NAFLD; obesity; type-2 diabetes mellitus; GLykLich diet; visceral adipose tissue; gut microbiota; whole foods; omega-3 fatty acids; macronutrients; micronutrients

Non-alcoholic fatty liver disease (NAFLD) is an increasing health problem worldwide [1,2]. Usually associated with insulin resistance, increased visceral fat mass and cardiovascular problems are referred to as metabolic-associated fatty liver disease (MAFLD) [1,3]. Such metabolic alterations are preventable and treatable through lifestyle changes such as diet. Currently, there are no common dietary recommendations for NAFLD therapy and associated co-morbidities. Here, I propose an anti-inflammatory and low glycemic diet, namely, the GLykLich diet.

Approximately $90 \%$ of patients with obesity and $60 \%$ of patients with type- 2 diabetes mellitus (T2DM) have some degree of NAFLD [4,5]. The prevalence of NAFLD correlates with BMI: $25 \%\left(B M I<25 \mathrm{~kg} / \mathrm{m}^{2}\right), 67 \%\left(B M I 25-30 \mathrm{~kg} / \mathrm{m}^{2}\right)$, and 91\% (BMI $\left.\geq 30 \mathrm{~kg} / \mathrm{m}^{2}\right)$ [5]. NAFLD increases the risk of T2DM as well as cardiovascular diseases (CVD) because in individuals with NAFLD, insulin is unable to normally suppress the production of glucose and very low lipoproteins (VLDL), leading to hyperglycemia and dyslipidemia [3]. In turn, the pancreatic $\beta$-cells attempt to compensate for the rising glucose levels, which results in hyperinsulinemia.

Silent inflammation - a low grade systemic chronic inflammation-is the common etiology of all metabolic derangements and is triggered by an unhealthy lifestyle including chronic stress, an obesogenic environment as well as a Western pattern diet. Thus, it contributes to the development and progression of NAFLD. Silent inflammation and 
insulin resistance are considered the main reasons for the abnormal accumulation of fat in the liver $[3,6]$.

The diet plays a pivotal role, and an unhealthy diet is associated with all the above-mentioned lifestyle diseases; certain nutrients may contribute to inflammatory processes [1,2,7-10]. In a study with 375 Iranian adults, the dietary pattern and risk for the development of NAFLD was assessed. The authors found that a Western pattern diet (consisting mainly of soft drinks, processed meats, high-fat dairy products, hydrogenated fats, refined grains and sugary sweets) was significantly associated with a higher risk of developing NALFD [1]. In a 1995 study of Australian adolescents assessed at 14 and 17 years of age $(n=995)$, a Western pattern diet was associated with an increased incidence of NAFLD [9]. This mainly concerns the high consumption of red and processed meats, refined carbohydrates, high intake of saturated fats and industry oils as well as food additives and conservatives. Such "inflammatory" nutrients are lighting the fire for lowgrade inflammation $[1,2,11]$.

If such inflammatory nutrients are consumed daily and are accompanied by other risk factors such as lack of movement and sleep, together with chronic stress and regular alcohol or nicotine consumption, then further metabolic and hormonal alterations may occur. Such a combination initiates the accumulation of visceral fat mass, gut dysbiosis, and leaky gut. Herein, the adipose tissue and the gut feed the circulation with inflammatory markers such as adipokines and cytokines as well as endotoxin and other inflammatory bacterial by-products, stoke the fire of the inflammatory process.

The visceral adipose tissue releases adipokines such as leptin and fat-specific cytokines such as resistin, chemerin, and IL-6, which are associated with insulin resistance and aberrant fat accumulation within the liver [12]. Gut microbiome is another rich source of inflammatory mediators such as lipopolysaccharides (LPS), also termed as endotoxins [13]. Deeper insights in the bacterial population have been gained during the last decade due to advances in biochemical sequencing methods. Researchers have identified a specific gut microbial signature related to NAFLD [13-15] by which specific bacteria strains are more abundant (Escherichia coli, Cutibacterium acnes) or less abundant (Clostridium coccoides, Bacteroides fragilis) in patients with metabolic related fatty liver [16]. An altered microbiome comprising the overgrowth of pathogenic gut bacteria (referred as dysbiosis) may disrupt gut integrity and trigger the loosening of the tight junctions. Hence, toxic and inflammatory substances released by pathogenic bacteria such as endotoxin are now able to pass the permeable gut barrier and enter circulation. Endotoxin activates Kupffer cells, liver-resident macrophages, and therefore triggers inflammation and liver injury $[13,14]$. Endotoxemia has been found to be positively correlated with both insulin resistance and histological severity of NAFLD [13]. Therefore, extensive visceral adipose tissues and altered gut are the two major sources for systemic inflammation.

Insulin resistance, the pathophysiological hallmark of NAFLD, is reversible through simple lifestyle intervention. Several studies have shown that a low carb diet or a ketogenic diet is effective in increasing insulin sensitivity and prevent further progression in a fully developed T2DM [2,3]. Overall, a diet that is low in refined grains, free sugars, and high in fiber and complex carbohydrates from wholegrains and starchy vegetables will balance insulin and blood glucose. In addition, such low-glycemic diets feed healthbeneficial bacteria such as Lactobacillus, Bifidobacterium, Clostridium, and Akkermansia, which have been shown to suppress pathogenic bacteria, provide energy for enterocytes, support gut lining, and support mucus production partly through the production of short chain fatty acids (SCFAs). SCFAs are able to enter circulation and act on distinct tissues (e.g., liver, adipose, muscle, and brain) by reducing inflammation, improving insulinsensitivity, inhibiting fatty acids synthesis and supporting detoxification [17,18].

Diet seems the most effective approach to quell the inflammatory fire, raising the question of whether conservative therapy would halt or even reverse the progression of fatty liver disease. So far, there are no common guidelines or diet recommendations suitable for the therapy of patients with NAFLD. NAFLD and healthy eating habits are interrelated 
through systemic inflammation and metabolism as well as through therapeutic dietary interventions for weight loss. Various diet schemas have been proposed in hepatic steatosis such as the Mediterranean diet $[19,20]$. The Mediterranean diet is recognized as effective and is linked to reduced risk of cardiometabolic diseases. This is partly explained by the effects of olive oil, nuts and seeds, legumes, and vegetables as well as fish, which are high in fiber, unsaturated fatty acids, and secondary plant substances having anti-inflammatory and anti-oxidative potential $[2,21]$.

Taking this one step further, the GLykLich diet consists of natural unprocessed foods, complex carbohydrates (fiber), high quality protein, anti-inflammatory fats, and is rich in secondary plant products. Altogether, these nutrients reduce de novo lipogenesis, improve insulin sensitivity, increase satiety, and modulate gut microbiota as well as reduce oxidative and inflammatory processes $[2,14,22,23]$. Thus, the GLykLich diet may attenuate the onset and the development of NAFLD.

The GLykLich diet is low-glycemic, which limits foods produced of refined grains and excludes simple sugars such as isolated fructose and glucose. High-glycemic index food causes insulin spikes and thus increases in parallel inflammation and de novo lipogenesis, which produce saturated fat from sugars and contributes to the abnormal fat accumulation within hepatocytes. In contrast, dietary fiber is inversely associated with risk of NAFLD with beneficial effects on fat and cholesterol digestion and modulation of the gut microbiome [3]. Dietary impact on modification of the intestinal microbiota has an influence on the regulation of the immune system [1].

Unexpected results have been presented by a recent study. The authors proposed a dual mechanism by which fructose, on one hand, induces a transcriptional program in hepatocytes driving de novo lipogenesis. On the other hand, fructose is metabolized by specific gut microbes providing acetate to feed hepatic fatty acid synthesis. Noteworthy, the microbiome produces, in addition to acetate, other SCFAs such as butyrate and propionate. To which extent these SCFAs contribute to lipogenic processes in hepatocytes remains to be determined.

Other studies performed in patients with obesity compared a low-fat high-carbohydrate diet and a high-fat low-carbohydrate diet in terms of intrahepatic liver fat. A meta-analysis (including 11 studies in 480 individuals that examined data from both MRI and changes in liver enzymes as a marker of liver health) concluded that a carbohydrate restriction is not efficient if it occurs at the expense of increased fat intake [3]. That being said, it is not the macronutrients, but rather the total energy intake that accounts for dietary success to reduce de novo lipogenesis, intrahepatic triglycerides, 24-h plasma glucose, and insulin concentrations in individuals with NAFLD [3].

It is of note that the quality of fat is not accounted for in most studies. Several researchers have highlighted the effect of fat quality $[3,24,25]$, which supports the view that only saturated fat impairs insulin sensitivity, whereas diets high in mono- and polyunsaturated fatty acids enhance insulin sensitivity [3]. The consumption of saturated fat mostly found in meat, dairy, and processed food is higher in most European countries than the recommended maximum of 10\% by national guidelines [3]. In 2018 and 2019, population studies confirmed that high consumption of red and processed meat correlates with the risk of NAFLD [2,21]. The quality of fat, meaning the degree of saturation of fatty acids, determines the anti-inflammatory effect of the fat and therefore the risk of NAFLD.

Mono-unsaturated (MUFA) as well as polyunsaturated fatty acids (PUFA) exert antiinflammatory functions. Olive oil, which is widely used in the Mediterranean diet, has high concentrations of the MUFA oleic acid. In addition, olive oil is rich in phenolic secondary plant substances such as tyrosol or oleuropin. In addition to anti-inflammatory properties, these bioactive compounds have been shown to exert a spectrum of health benefits such as cardioprotective, anti-oxidative, anti-cancerous, or act as regulators of the intestinal microbiota [26].

Nuts, seeds, and their oils are good sources for the PUFA omega- 3 fatty acids, in particular alpha-linolic acid (ALA). Two other omega-3 fatty acids are EPA (eicosapentaenoic 
acid) and DHA (docosahexaenoic acid), which are mainly found in fatty fish such as salmon, tuna, mackerel, and herring. Of note, EPA and DHA are precursors for resolvins, which bring about a programmed resolution of the inflammatory process [27]. DHA, in particular, serves as the precursor for the synthesis of protectins that have anti-inflammatory and neuroprotective activities [27]. Although ALA may be converted to EPA and DHA, only a small percentage is being converted, with a rate of about $5 \%$ to EPA and less than $1 \%$ to DHA [27]. Due to this low rate of conversion of ALA to EPA and DHA, and a generally low consumption of these omega-3 fatty acids, most people, in general, are deficient in EPA or DHA. It is therefore not surprising that patients with NAFLD have low concentrations of EPA and DHA [25]. NAFLD in humans and preclinical mouse models confirm a decline of PUFA in blood and liver tissue $[25,28]$. Recent meta-analyses and clinical trials confirm that the supplementation of EPA and DHA improves liver health by reducing steatosis [28]. A study with 19 healthy men who received an omega-3 fatty acid supplement $4 \mathrm{~g}$ per day) for eight weeks compared with the baseline, significantly ameliorated fasting and postprandial hepatic de novo lipogenesis and increased fatty acid oxidation [3].

In mice, it was shown that high-fat feeding increases the proportion of endotoxincontaining gut bacteria and induces endotoxaemia, inflammation, insulin resistance, and hepatic steatosis [3]. These changes were induced by feeding the mice saturated but not polyunsaturated fat [29], and were attenuated after antibiotic treatment [30]. This suggests that saturated fat intake, gut microbiota, and metabolic inflammation are causally linked. Similar results were shown in human studies: 38 healthy overweight adults were treated for three weeks with either saturated or unsaturated fat [24]. Only the saturated fatenriched diet induced endotoxaemia, adipose tissue inflammation, and insulin resistance. In addition to saturated fat, fructose intake has been shown to increase bacterial endotoxin concentrations and markers of liver injury in rodents, non- human primates, and humans [3]. This suggests that the quality of macronutrients consumed determines the risk of NALFD.

To date, there is no approved pharmacotherapy; the present therapeutic cornerstone for non-alcoholic fatty liver diseases (NAFLD) is a lifestyle intervention with a focus on diet [2]. There is no single nutrient to prevent the progression of NAFLD, rather, it is the complexity of substances in whole unprocessed foods that reduce inflammatory processes, improve metabolic state, and thus reverse NAFLD. Micronutrients such as vitamins, minerals, and secondary plant substances are important cofactors and regulators for several metabolic reactions as well as inflammatory processes. It is thus critical to ensure our food provides enough of those power substances. Focus should be paid to the grade of food processing and livestock farming, which may determine the amount of anti-inflammatory and anti-oxidative nutrients. Animal-based food such as lean beef, chicken, and fish are good sources of B-vitamins (i.p., vitamin B12, B3). Vitamins are essential micronutrients in the maintenance of health, which cannot be synthesized sufficiently from other molecules alone. Recent studies have highlighted the importance of dietary vitamin composition and liver fat accumulation [10,31]. Vitamins regulate several enzymatic processes in the liver, and alterations in vitamin metabolism are believed to play a critical role in NAFLD progression. Vitamin $\mathrm{C}$ and $\mathrm{E}$ exert anti-oxidant activities and have been attributed to mitigate hepatocyte injury [32]. Alterations in serum levels of vitamin D, vitamin B12, B3 (niacin), and folate are strongly associated with NAFLD severity [32]. Vitamin B3 is a precursor of the coenzyme nicotinamide adenine dinucleotide and nicotinamide adenine dinucleotide phosphate (NADH/NADPH), which plays an important role in lipid metabolism. Niacin treatment on dyslipidemic patients reduces both the plasma triglyceride concentration and the hepatic fat content, and ameliorates liver enzymes such as hepatic transaminase [33]. The liver is an important storage site of vitamin B12, which plays an important role in DNA synthesis and repair. Low vitamin B12 in the diet of pregnant women induces higher rates of adiposity and T2DM in their subsequent offspring and is accompanied by changes in hepatic gene expression involved in lipid metabolic pathways. These alterations could be reversed in the offspring through vitamin B12 reconstitution [34]. 
Similar to vitamins, changes in mineral concentrations are correlated with the development of NAFLD (i.e., zinc, iodide, and selenium). Zinc has important immunomodulatory functions and supports anti-oxidative reactions. Moreover, it is integrated in the metabolism of insulin (synthesis, storage, secretion and signaling). In rodents, zinc deficiency augments oxidative stress in hepatocytes and is stimulated with aberrant accumulation of lipids within liver tissue [35]. However, no profound clinical study has been performed to show the direct implications of zinc in the development of NAFLD in humans.

Iodide and selenium are critical minerals for thyroid hormone metabolism. Hypothyroidism is associated with obesity and knowing that over $60 \%$ percent of obese patients have NAFLD, it might be possible that these two minerals are likely critical to liver metabolism. The liver synthesizes and secretes selenoprotein P (SELENOP), a glycoprotein transporting selenium from the liver to other tissues. Selenium is an important building component of the selenium-dependent enzymes, deiodinases, which convert inactive T4 to the active T3. In addition, selenium also plays an important role for other enzymes within the redox-system and its deficiency increases the secretion of cytokines, which contribute to inflammatory processes within the body.

Secondary plant substances comprise different classes of phytochemicals with antioxidant and anti-inflammatory activities [36,37]. These include polyphenols, carotenes, phytosterols, and phytostanols and are largely found in fruits, vegetables, grains, nuts, seeds, and legumes [10]. A high and varied intake of such phytochemicals seems to ameliorate hepatic steatosis, oxidative stress, inflammation, and gut dysbiosis. Clinical trials have confirmed the beneficial effects of plant-based foods, which are rich in bioactive phytochemicals, on patients with fatty liver diseases [7,10,36].

Overall, a micronutrient rich diet benefits liver metabolism and thus may lower the risk of developing NAFLD. An altered liver metabolism may, in turn, limit the absorption as well as the homeostasis of micronutrients. First, hepatocytes synthesize binding, transport, and regulatory proteins required for micronutrient homeostasis. Second, the absorption of fat-soluble vitamins and some bioactive phytochemicals requires bile, which is produced by hepatocytes. Thus, a well-functioning liver is important for the absorption, metabolism, and homeostasis of micronutrients.

To summarize, an excessive macronutrient intake of saturated fats and sugar, in particular, contributes to hepatic injury and perturbations of energy homeostasis. In addition, the deficient intake of micronutrients exacerbates these problems by deregulating lipid homeostatic, anti-oxidant, and anti-inflammatory pathways. At first glance, it seems simple to recommend an anti-NALFD diet by simply reducing the number of calories (macronutrient intake) and supplement with micronutrients. However, the reality is more challenging, because the human diet is complex, and each food constitutes a specific matrix including different essential, semi-essential, and non-essential nutrients interacting with each other. Therefore, the use of isolated nutrients is not the full solution for the therapy of NAFLD. It is my firm view that the best diet to treat NAFLD is an anti-inflammatory low glycemic GLykLich diet. This diet includes a spectrum of unprocessed whole-foods, both seasonal and regional. Plant-based foods should be the focus, supplemented with fish, grass-fed lean meat, poultry, and dairy. Such a diet will ensure the sufficient uptake of fiber, good-quality protein, and healthy fats as well as a panel of micronutrients. It will also support the microbiota and prevent visceral fat deposition and therefore decrease the risk for NAFLD. The GLykLich diet should be considered key in the therapy for NALFD and related co-morbidities such as obesity and T2DM.

Funding: This research received no external funding.

Institutional Review Board Statement: Not applicable.

Informed Consent Statement: Not applicable.

Data Availability Statement: Not applicable.

Conflicts of Interest: The author declares no conflict of interest. 


\section{References}

1. Salehi-Sahlabadi, A.; Sadat, S.; Beigrezaei, S.; Pourmasomi, M.; Feizi, A.; Ghiasvand, R.; Hadi, A.; Clark, C.C.T.; Miraghajani, M. Dietary patterns and risk of non-alcoholic fatty liver disease. BMC Gastroenterol. 2021, 21, 41. [CrossRef]

2. Houttu, V.; Csader, S.; Nieuwdorp, M.; Holleboom, A.G.; Schwab, U. Dietary Interventions in Patients with Non-alcoholic Fatty Liver Disease: A Systematic Review and Meta-Analysis. Front. Nutr. 2021, 8, 716783. [CrossRef]

3. Yki-Järvinen, H.; Luukkonen, P.K.; Hodson, L.; Moore, J.B. Dietary carbohydrates and fats in nonalcoholic fatty liver disease. Nat. Rev. Gastroenterol. Hepatol. 2021, 18, 770-786. [CrossRef]

4. Divella, R.; Mazzocca, A.; Daniele, A.; Sabbà, C.; Paradiso, A. Obesity, Nonalcoholic Fatty Liver Disease and Adipocytokines Network in Promotion of Cancer. Int. J. Biol. Sci. 2019, 15, 610-616. [CrossRef]

5. Younossi, Z.; Tacke, F.; Arrese, M.; Sharma, B.C.; Mostafa, I.; Bugianesi, E.; Wong, V.W.-S.; Yilmaz, Y.; George, J.; Fan, J.; et al. Global Perspectives on Nonalcoholic Fatty Liver Disease and Nonalcoholic Steatohepatitis. Hepatology 2019, 69, 2672-2682. [CrossRef]

6. Hotamisligil, G.S. Inflammation and metabolic disorders. Nature 2006, 444, 860-867. [CrossRef]

7. Li, H.Y.; Gan, R.Y.; Shang, A.; Mao, Q.Q.; Sun, Q.C.; Wu, D.T.; Geng, F.; He, X.Q.; Li, H.B. Plant-Based Foods and Their Bioactive Compounds on Fatty Liver Disease: Effects, Mechanisms, and Clinical Application. Oxid. Med. Cell. Longev. 2021, 2021, 6621644. [CrossRef]

8. Moore, J.B.; Fielding, B.A. Sugar and metabolic health: Is there still a debate? Curr. Opin. Clin. Nutr. Metab. Care 2016, 19, 303-309. [CrossRef]

9. Oddy, W.H.; Herbison, C.E.; Jacoby, P.; Ambrosini, G.L.; O'Sullivan, T.A.; Ayonrinde, O.T.; Olynyk, J.K.; Black, L.J.; Beilin, L.J.; Mori, T.A.; et al. The Western Dietary Pattern Is Prospectively Associated with Nonalcoholic Fatty Liver Disease in Adolescence. Am. J. Gastroenterol. 2013, 108, 778-785. [CrossRef]

10. Pickett-Blakely, O.; Young, K.; Carr, R.M. Micronutrients in Nonalcoholic Fatty Liver Disease Pathogenesis. Cell. Mol. Gastroenterol. Hepatol. 2018, 6, 451-462. [CrossRef]

11. Jegatheesan, P.; De Bandt, J. Fructose and NAFLD: The Multifaceted Aspects of Fructose Metabolism. Nutrients 2017, 9, 230. [CrossRef]

12. Taylor, E.B. The complex role of adipokines in obesity, inflammation, and autoimmunity. Clin. Sci. 2021, 135, 731-752. [CrossRef]

13. Park, E.; Jeong, J.-J.; Won, S.-M.; Sharma, S.P.; Gebru, Y.A.; Ganesan, R.; Gupta, H.; Suk, K.T.; Kim, D.J. Gut Microbiota-Related Cellular and Molecular Mechanisms in the Progression of Nonalcoholic Fatty Liver Disease. Cells 2021, 10, 2634. [CrossRef]

14. De Oca, A.P.-M.; Julián, M.T.; Ramos, A.; Puig-Domingo, M.; Alonso, N. Microbiota, Fiber, and NAFLD: Is There Any Connection? Nutrients 2020, 12, 3100. [CrossRef]

15. Wang, T.; Guo, X.-K.; Xu, H. Disentangling the Progression of Non-alcoholic Fatty Liver Disease in the Human Gut Microbiota. Front. Microbiol. 2021, 12, 728823. [CrossRef]

16. Aron-Wisnewsky, J.; Vigliotti, C.; Witjes, J.; Le, P.; Holleboom, A.G.; Verheij, J.; Nieuwdorp, M.; Clément, K. Gut microbiota and human NAFLD: Disentangling microbial signatures from metabolic disorders. Nat. Rev. Gastroenterol. Hepatol. 2020, 17, 279-297. [CrossRef]

17. Visekruna, A.; Luu, M. The Role of Short-Chain Fatty Acids and Bile Acids in Intestinal and Liver Function, Inflammation, and Carcinogenesis. Front. Cell Dev. Biol. 2021, 9, 703218. [CrossRef]

18. Salles, B.I.M.; Cioffi, D.; Ferreira, S.R.G. Probiotics supplementation and insulin resistance: A systematic review. Diabetol. Metab. Syndr. 2020, 12, 98. [CrossRef]

19. Abenavoli, L.; Boccuto, L.; Federico, A.; Dallio, M.; Loguercio, C.; Di Renzo, L.; De Lorenzo, A. Diet and Non-Alcoholic Fatty Liver Disease: The Mediterranean Way. Int. J. Environ. Res. Public Health 2019, 16, 3011. [CrossRef]

20. Di Renzo, L.; Cioccoloni, G.; Falco, S.; Abenavoli, L.; Moia, A.; Salimei, P.S.; De Lorenzo, A. Influence of FTO rs9939609 and Mediterranean diet on body composition and weight loss: A randomized clinical trial. J. Transl. Med. 2018, 16, 308. [CrossRef]

21. Zadeh, S.H.; Mansoori, A.; Hosseinzadeh, M. Relationship between dietary patterns and non-alcoholic fatty liver disease: A systematic review and meta-analysis. J. Gastroenterol. Hepatol. 2021, 36, 1470-1478. [CrossRef] [PubMed]

22. Zhao, H.; Yang, A.; Mao, L.; Quan, Y.; Cui, J.; Sun, Y. Association Between Dietary Fiber Intake and Non-alcoholic Fatty Liver Disease in Adults. Front. Nutr. 2020, 7, 593735. [CrossRef] [PubMed]

23. Gupta, A.; Pandey, A.K. Nutraceuticals and Natural Product Pharmaceuticals; Elsevier: Amsterdam, The Netherlands, 2019; pp. 71-104.

24. Luukkonen, P.K.; Sädevirta, S.; Zhou, Y.; Kayser, B.; Ali, A.; Ahonen, L.; Lallukka, S.; Pelloux, V.; Gaggini, M.; Jian, C.; et al. Saturated Fat Is More Metabolically Harmful for the Human Liver Than Unsaturated Fat or Simple Sugars. Diabetes Care 2018, 41, 1732-1739. [CrossRef] [PubMed]

25. Spooner, M.H.; Jump, D.B. Omega-3 fatty acids and nonalcoholic fatty liver disease in adults and children: Where do we stand? Curr. Opin. Clin. Nutr. Metab. Care 2019, 22, 103-110. [CrossRef] [PubMed]

26. Finicelli, M.; Squillaro, T.; Galderisi, U.; Peluso, G. Polyphenols, the Healthy Brand of Olive Oil: Insights and Perspectives. Nutrients 2021, 13, 3831. [CrossRef]

27. Maciejewska-Markiewicz, D.; Stachowska, E.; Hawryłkowicz, V.; Stachowska, L.; Prowans, P. The Role of Resolvins, Protectins and Marensins in Non-Alcoholic Fatty Liver Disease (NAFLD). Biomolecules 2021, 11, 937. [CrossRef] 
28. Capanni, M.; Calella, F.; Biagini, M.R.; Genise, S.; Raimondi, L.; Bedogni, G.; Svegliati-Baroni, G.; Sofi, F.; Milani, S.; Abbate, R.; et al. Prolonged n-3 polyunsaturated fatty acid supplementation ameliorates hepatic steatosis in patients with non-alcoholic fatty liver disease: A pilot study. Aliment. Pharmacol. Ther. 2006, 23, 1143-1151. [CrossRef]

29. Caesar, R.; Tremaroli, V.; Kovatcheva-Datchary, P.; Cani, P.D.; Bäckhed, F. Crosstalk between Gut Microbiota and Dietary Lipids Aggravates WAT Inflammation through TLR Signaling. Cell Metab. 2015, 22, 658-668. [CrossRef]

30. Cani, P.D.; Bibiloni, R.; Knauf, C.; Waget, A.; Neyrinck, A.M.; Delzenne, N.M.; Burcelin, R. Changes in Gut Microbiota Control Metabolic Endotoxemia-Induced Inflammation in High-Fat Diet-Induced Obesity and Diabetes in Mice. Diabetes 2008, 57, 1470-1481. [CrossRef]

31. Winters-van Eekelen, E.; Verkouter, I.; Peters, H.P.; Alssema, M.; de Roos, B.G.; Schrauwen-Hinderling, V.B.; Roumans, K.H.M.; Schoones, J.; Zock, P.L.; Schrauwen, P.; et al. Effects of dietary macronutrients on liver fat content in adults: A systematic review and me-ta-analysis of randomized controlled trials. Eur. J. Clin. Nutr. 2021, 75, 588-601. Available online: https: //www.nature.com/articles/s41430-020-00778-1 (accessed on 8 November 2021). [CrossRef]

32. Raza, S.; Tewari, A.; Rajak, S.; Sinha, R.A. Vitamins and non-alcoholic fatty liver disease: A molecular insight. Liver Res. 2021, 5, 62-71. [CrossRef] [PubMed]

33. Hu, M.; Chu, W.; Yamashita, S.; Yeung, D.K.W.; Shi, L.; Wang, D.; Masuda, D.; Yang, Y.; Tomlinson, B. Liver fat reduction with niacin is influenced by DGAT-2 polymorphisms in hypertriglyceridemic patients. J. Lipid Res. 2012, 53, 802-809. [CrossRef] [PubMed]

34. Deshmukh, U.; Katre, P.; Yajnik, C.S. Influence of Maternal Vitamin B12and Folate on Growth and Insulin Resistance in the Offspring. Prev. Asp. Early Nutr. 2013, 74, 145-156. [CrossRef]

35. Carr, R.M.; Correnti, J. Insulin resistance in clinical and experimental alcoholic liver disease. Ann. N. Y. Acad. Sci. 2015, 1353, 1-20. [CrossRef] [PubMed]

36. Makhmudova, U.; Schulze, P.C.; Lütjohann, D.; Weingärtner, O. Phytosterols and Cardiovascular Disease. Curr. Atheroscler. Rep. 2021, 23, 68. [CrossRef] [PubMed]

37. Nattagh-Eshtivani, E.; Barghchi, H.; Pahlavani, N.; Barati, M.; Amiri, Y.; Fadel, A.; Khosravi, M.; Talebi, S.; Arzhang, P.; Ziaei, R.; et al. Biological and pharmacological effects and nutritional impact of phytosterols: A comprehensive review. Phytother. Res. 2021, 36, 299-322. [CrossRef] 